Rapid Reviews COVID-19

\title{
Review 2: "The infection fatality rate of COVID-19 inferred from seroprevalence data"
}

Kenji Mizumoto ${ }^{1}$, Gerardo Chowell ${ }^{2}$

${ }^{1}$ Graduate School of Advanced Integrated Studies in Human Survivability, Kyoto University, ${ }^{2}$ Department of Population Health Sciences, Georgia State University School of Public Health

Published on: Aug 23, 2020

DOI: $10.1162 / 2 \mathrm{e} 3983 f 5.8257 \mathrm{bfb} 2$

License: Creative Commons Attribution 4.0 International License (CC-BY 4.0). 


\section{$\underline{\text { RR:C19 Evidence Scale rating by reviewer: }}$}

- Potentially informative. The main claims made are not strongly justified by the methods and data, but may yield some insight. The results and conclusions of the study may resemble those from the hypothetical ideal study, but there is substantial room for doubt. Decision-makers should consider this evidence only with a thorough understanding of its weaknesses, alongside other evidence and theory. Decisionmakers should not consider this actionable, unless the weaknesses are clearly understood and there is other theory and evidence to further support it.

*****************************************

\section{Review:}

In this paper, the author analyzes and synthesizes estimates of the infection fatality rate of COVID-19 based on data reported in seroprevalence studies. We recommend publication once the following comments are appropriately addressed:

1) We strongly recommend excluding papers retrieved from preprint servers.

2) For example, we are familiar with the following Japanese studies cited as refs. 11 and 29, and we believe these studies should be excluded from the analysis. Specifically, ref. 11 has low external validity because medical facilities are not "outpatient clinics" but outpatients that attend a tertiary hospital (this study has been criticized in Japan for this reason). Similarly, for the study reported in ref. 29, the authors recruited participants via the Internet. This leads to a critical selection bias and low external validity (generalization).

Hence, this indicates that preprint studies which have not gone through peer review should be excluded from the analysis.

11. Doi A, Iwata Kuroda H, Hasuike T, Nasu S, Kanda A, et al. Seroprevalence of novel coronavirus disease (COVID-19) in Kobe, Japan. medRxiv

https://doi.org/10.1101/2020.04.26.20079822

"Randomly selected patients who visited outpatient clinics and received blood testing for any reason. Patients who visited the emergency department or the designated fever consultation service were excluded."

29. Takita M, Matsumura T, Yamamoto K, Yamashita E, Hosoda K, Hamaki T, Kusumi E. 
Regional difference in seroprevalence of SARS-CoV-2 in Tokyo: Results from the community point-of-care antibody testing. medRxiv 2020.06.03.20121020; doi: https://doi.org/10.1101/2020.06.03.20121020

"Two community clinics located in the major railway stations in Tokyo (Navitas Clinic Shinjuku and Tachikawa)"

3) It would be helpful to provide a flow diagram of the study selection process in order to clarify the selection criteria. 